\title{
A Novel Approach to Segmentation of Persian Cursive Script Using Decision Tree
}

\author{
Shahriar Pirnia Naeini, Maryam Khademi, and Alireza Nikookar
}

\begin{abstract}
In this paper, we propose a novel method for segmentation of online Persian handwriting into the fundamental building blocks of Persian letters. Employing the findings of our previous work to determine the segmentation points of cursive words and applying some smoothing techniques to improve our results, we have advanced our model to form the pre-segments into the predefined building blocks (BBs) which will be used later for recognizing letters in written words. We have utilized a decision tree to accomplish this task and the $98.6 \%$ accuracy has been obtained in forming the BBs as the overall result.
\end{abstract}

Index Terms-Decision tree, feature extraction, online cursive script, Persian words, segmentation.

\section{INTRODUCTION}

In the research area of handwriting recognition, the problem of segmenting Persian cursive script has been considered quite recently [1]-[3]. The cursive nature of Persian script and the existence of different handwriting styles for its alphabet as well as different forms of each letter, depending on its location in a word, which make segmentation and recognition of Persian words a challenging task [1], [2], [4], [5]. In addition, diacritics as ordinary parts of most Persian letters, having various styles and forms in different places in words, make the task really complex [4], [5]. There are a number of techniques for reducing the complexity and difficulties of the process [6], one of which is segmentation.

In segmentation approach [2], [3], [7], the written word breaks down into smaller parts called segments. These parts will be used later to recognize the letters which form the written word. Although breaking down a word into segments looks easy, finding the right points for an exact separation is a difficult task. In [3], we have accomplished this job; however in order to proceed with the process, the segments must be formed into some predefined building blocks, which will be identified later as letters of the written word. Therefore, in

Manuscript received March 7, 2012; revised May 6, 2012.

It is pleasure to acknowledge the financial support of South Tehran Branch, Islamic Azad University, since this investigation has accomplished in the form of a research plan named "Providing a new feature-based method for online segmentation of Persian words" with encouragement of South Tehran Branch, Islamic Azad University.

Shahriar Pirnia Naeini is with the Department of Computer Science, South Tehran Branch, Islamic Azad University, Tehran 11365/4435, Iran (e-mail: sh_pirnia@azad.ac.ir).

Maryam Khademi is with the Department of Applied Mathematics, South Tehran Branch, Islamic Azad University, Tehran 11365/4435, Iran (e-mail: khademi@azad.ac.ir).

Alireza Nikookar is with the Young Researchers Club, South Tehran Branch, Islamic Azad University, Tehran 11365/4435, Iran (e-mail: a_nikookar@azad.ac.ir). this paper, we have proposed a novel method of forming predefined building blocks from segments, which will ease the process of Persian handwriting recognition.

The rest of the paper is organized as follows: in section 2, pre-segmentation process is discussed. In section 3, forming the building blocks from segments using decision tree is presented. In section 4, experimental results are shown and finally the paper will be ended in section 5 by conclusions and future works.

\section{Pre-Segmentation}

Regarding cursive handwriting, the objective of the pre-segmentation process is to divide written words into segments containing at most one letter [7], in other words, pre-segmentation is the operation of setting some points on cursive handwriting so that between each two successive points there will be at most one character. Thus, a segment is exactly explained as the set of points between each pair of successive segmentation points [7].

\section{A. Features}

In order to find the segmentation points in a written word, we used six features of points of writing path, which are as follows: direction of writing, sequence of directions, starting and ending points of strokes, the last repeated point in each cluster of adjacent points, right and left derivatives in a point, and the second derivative [3]. Using these features and combination of them, we are able to distinguish the segmentation points of written words. Later, we used these points and segments between them to form predefined building blocks.

\section{B. Smoothing}

In [3], we mentioned that there was still a possibility of improving the result of pinpointing the segmentation points. One approach to obtain better results is to reduce the noise of the written word's curves. In order to do that, we apply an average filter to the curve to smooth it and accordingly improve the result of the pinpointing process. Consequently, the segmentation points will be more accurate while irrelevant points will be reduced to some extent.

\section{Segmentation Using Decision Tree}

In the previous section, we have discussed some features to pinpoint segmentation points in the input, which were leaded to segments that will help us later to recognize letters of the written word. We also mentioned that we applied an average filter to improve the pinpointing process, but before 
jumping to recognition step, we need to make sure that each two segmentation points and respectively the segment between them represent a letter.

To do so, we have to examine each segment and check if it needs to be combined with other segments in order to form a new segment which represents a letter. Since we do not know what the written word is and therefore what its constituent letters are, we need to introduce some predefined forms which we term "Basic Building Blocks" to form segments into these building blocks. Then we use them to recognize the constituent letters and consequently the written word.

\section{A. Basic Building Blocks}

As mentioned earlier, we need to define some forms as building blocks to help us to refine the segments which have been detected in the previous stage. After examining Persian letters, 15 blocks were identified, which help us to form the body of any Persian letter. Some of these blocks are shown in Fig. 1.

Using these building blocks and combining them, we can recognize the bodies of letters, but before that we need to examine the detected segments and combine them to form building blocks if necessary. We will use a decision tree classifier as a means of doing so.

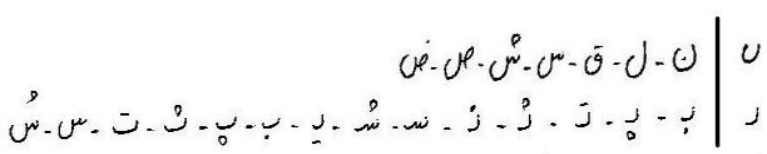

Fig. 1. Two examples of Building Blocks on the right side and letters on the left

\section{B. Decision Tree}

A decision tree is a tree-like graph which consists of internal and leaf nodes and branches [8]. One of the uses of decision trees is to classify patterns in right categories. In order to do so, we put a primary condition with respect to the classifying problem in a root node and then we establish a tree using internal nodes, branches and leaves. Each internal node represents a conditional test according to other parameters of the problem and the result of the test goes through a branch to another internal node or a leaf, which is a category or class to which testing pattern belongs. A typical decision tree has been shown in Fig. 2 [8]:



Fig. 2. A typical decision tree

\section{Forming Building Blocks}

After detecting segments in the written word and putting them in a sorted list (based on detecting order), we use a decision tree to form building blocks. In order to do that, we start to examine each segment in the list to see whether or not it matches with a predefined block. If it does, we will remove it from the list and put the respective block in another list. Otherwise we will pick next segment in the list and use a decision tree to classify the overall form created by those segments into one of the blocks. If a category is found, we will remove the segments and put the block which is formed by all segments in the decision path in the list. Otherwise we continue with the next segments until we find a block. We do the mentioned process for all the segments. The final list will contain all essential building blocks which we need to recognize the constituent letters of the written word.

It must be mentioned that there might be noise in the written word leading to extra segments which make the forming process problematic. In order to decrease the effect of these extra segments on the process, a backtracking approach will be employed. Indeed, a "non-block form" leaf is added to the end of each decision path and when the tree reaches this leaf, it replaces the last segments in its path with the next segment of the list and continues the decision process.

\section{EXPERIMENTAL RESULTS}

To evaluate the accuracy and efficiency of the proposed method, we developed an application using the C\#.NET and Microsoft Visual Studio. We also implemented an average filter to make writing curves smoother using MATLAB. In order to evaluate the method, 1000 handwritten cursive words were input to the application. The overall result of $98.6 \%$ has been gained for the sample pack. Table 1 shows more information for some of the words in the sample pack.

TABLE I: The Results OBtAined From The Proposed Algorithm

\begin{tabular}{|c|c|c|c|}
\hline Words & Total Number of BBs & $\begin{array}{l}\text { Number of BBs } \\
\text { correctly detected }\end{array}$ & $\begin{array}{l}\text { Number of } \\
\text { BBs wrongly } \\
\text { Detected }\end{array}$ \\
\hline سلام & 5 & 5 & 0 \\
\hline قسطنطنيه & 12 & 12 & 0 \\
\hline باغستان & 9 & 8 & 1 \\
\hline علبرضا & 6 & 5 & 1 \\
\hline جلجِر اغ & 7 & 6 & 1 \\
\hline كهكيلويه & 8 & 8 & 0 \\
\hline
\end{tabular}

As it has been shown in the Table 1, for some words containing certain letters, the method was unable to form all the anticipated building blocks completely; this particularly occurred to letters " $\varepsilon$ " and "غ்".

\section{CONCLUSIONS AND FUtURE WORKS}

In this paper, we have introduced a method of forming predefined building blocks from segmentation points and segments. These building blocks have been founded on an investigation of a wide range of Persian written words. The proposed method is based on decision tree algorithm and combines the segments to form fifteen predefined building blocks. It is simple and fast, furthermore, our experimental results showed $98.6 \%$ accuracy in forming building blocks of 1000 handwritten Persian cursive words. Therefore it is not only efficient but also accurate. These building blocks will be used later to identify letters in a written word.

Our method is an intermediate-phase approach to 
recognition of final words which increases the accuracy and efficiency as well as reducing the complexity of the process. Although a number of methods for recognition of online Persian words have been introduced in the literature recently, because of the novelty of the presented approach and the fact that it is an intermediate-phase method, we could not find other methods to make comparisons.

As a future work, we can focus on improving the final result of the proposed method by enhancing the decision criteria or using an extended version of decision tree such as a fuzzy decision tree. The results gained here can also be utilized in the next step for recognition of the whole written word.

\section{REFERENCES}

[1] R. Halavati, M. Jamzad, and M. Soleymani, "A novel approach to Persian online handwriting recognition," World Academy of Science, Engineering and Technology, vol. 11, pp. 81-85, 2005.

[2] S. Izadi, M. Haji, and C. Y. Suen, "A new segmentation algorithm for online handwritten word recognition in Persian script," in Proc. Eleventh International Conf. Frontiers in Handwriting Recognition (CFHR 2008), Montreal, Canada, 2008, pp. 598-603.

[3] S. Pirnia, M. Khademi, A. Nikookar, and Z. Bani, "A feature-based approach to segmentation of online Persian cursive script," in Proc. the 2010 International Conf. Computer and Software Modeling (ICCSM 2010), Manila, Philippines, 2010, pp. 224-228.

[4] Z. Al Aghbari and S. Brook, "A holistic paradigm for classifying and retrieving historical Arabic handwritten documents," Expert Systems with Applications, vol. 36, pp. 10942-10951, 2009.

[5] H. M. S. Beigi, K. Nathan, G. J. Clary, and J. Subrahmonia, "Challenges of handwriting recognition in Farsi, Arabic and other languages with similar writing style on online digit recognizer," in Proc. 2nd Annu. Conf. Technological advancements in developing countries, Columbia University, New York, 1994, pp. 23-24.

[6] M. Khademi, A. Nikookar, and A. Farahani, "Recognizing online Persian characters using feature extraction," in Proc. Electronics, Computers and Artificial Intelligence Conf. (ECAI 2009), Piteşti, Romania, 2009.

[7] J. Sternby, J. Morwing, J. Andersson, and Ch. Friberg, "On-line Arabic handwriting recognition with templates," Pattern recognition, vol. 42, pp. 3278-3286, December 2009.

[8] J. Han and M. Kamber, Data Mining: Concepts and Techniques, San Francisco: Morgan Kaufmann Publishers, 2006, pp. 291-296.

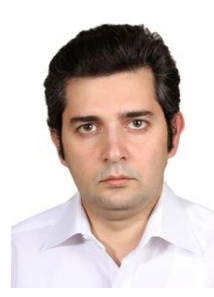

Shahriar Pirnia Naeini was born in 1971 in Isfahan, Iran. He received his B.Sc. and M.Sc. degrees from Tehran University, Tehran, Iran in 1995 and Islamic Azad University, South Tehran Branch, Tehran, Iran in 1999, respectively. He is a lecturer in Islamic Azad University, South Tehran Branch, Tehran, Iran. He was the Director of Internet Department of Islamic Azad University, South Tehran Branch. He has also worked as a Designer and Developer of Software Systems. He is the author of four papers in national or international journals or conferences and also collaborated in several research projects.

His current research interests include neural networks, handwriting recognition, artificial intelligence, image processing and data mining. He has been a faculty member of the deprtment of computer science in Islamic Azad University, South Tehran Branch since 2000.

Mr. Pirnia is a member of IACSIT.



Maryam Khademi was born in 1967 in Borujred, Iran. She received the B.Sc., M.Sc. and Ph.D degrees from Tarbiat Moaalem University in 1989, Iran University of Science and Technology in 1991, and University of Tehran in 2004, respectively. She is the author or coauthor of more than sixty-five articles in national or international journals and conferences proceedings and also collaborated in several research projects. She wrote a book entitled "Advanced Engineering Mathematics", 2011 and has cooperated in translating two books, namely "Calculus and Analytic Geometry, George Thomas Brinton", 2010 and "Research to Manuscript, Micheal Jay Katz", 2012. Her current research interests include finite group theory, graph theory, modeling and optimization, image processing, handwriting recognition, artificial intelligence and data mining She is a faculty member of the deprtment of applied mathematics in Islamic Azad University, Sorth Tehran Branch, since 1991.



Alireza Nikookar received his B.Sc. degree in computer engineering - software from Islamic Azad University - South Tehran Branch, Tehran, Iran in 2006 and his M.S degree in artificial intelligence from Islamic Azad University - Science and Research Campus, Tehran, Iran in 2010. He has been a researcher in the fields of Computational Intelligence and Recognition Systems at Islamic Azad University South Tehran Branch (IAU-STB) for 5 years and an invited lecturer at IAU-STB for 2 years. He was a user interface (UI) and user experience (UX) designer and consultant of several national projects.

$\mathrm{He}$ is currently working at Chargoon Company as UX evangelist and manager. His interests are in fields of Computational Intelligence, Human Computer Interactions, Information Visualization, Recognition Systems, Smart Environment, and Software Engineering.

Mr. Nikookar is a professional member of ACM and member of IACSIT. 